\title{
Electrochemical oxidation mechanism of guanine and adenine using a glassy carbon microelectrode
}

\author{
A.M. Oliveira-Brett*, V. Diculescu, J.A.P. Piedade \\ Departamento de Química, Faculdade de Ciências e Tecnologia, Universidade de Coimbra, 3004-535 Coimbra, Portugal
}

Received 1 June 2001; received in revised form 6 August 2001; accepted 5 September 2001

\begin{abstract}
The electrochemical oxidation mechanism of guanine and adenine was investigated using a glassy carbon microelectrode and cyclic and differential pulse voltammetry. It is $\mathrm{pH}$-dependent and the electron transfer process occurs in consecutive steps with the formation of strongly adsorbed dimers on the electrode surface for both compounds. (C) 2002 Elsevier Science B.V. All rights reserved.
\end{abstract}

Keywords: Electrochemical oxidation; Guanine; Adenine; Voltammetry

\section{Introduction}

The electrochemical mechanisms of guanine and adenine oxidation in solution have been thoroughly investigated, however, there have been few mechanistic studies of their oxidation at electrodes.

The purine bases, adenine and guanine, are involved in cellular energy transduction and signalling mediated by enzymatic oxidation reactions. Electrochemical processes involved in purine DNA base oxidation are similar to those involving enzymatic oxidation reactions and are of crucial importance to improve the interpretation of DNA drug/metal interactions that lead to oxidative damage of the heredity biomolecule [1-4]. The electrochemical oxidation mechanism of guanine and adenine using a carbon fibre microelectrode was investigated. The reduced capacitive contribution of microelectrodes makes it possible to study the electron transfer (ET) reactions of these processes by means of fast scan rate cyclic voltammetry and to detect more sensitive electrode processes by differential pulse voltammetry, allowing a better understanding of the ET mechanisms and adsorption phenomena.

\section{Experimental}

Adenine (6-aminopurine) and guanine (2-amino-6-hydroxypurine) were obtained from Sigma and used without

\footnotetext{
* Corresponding author. Tel./fax: +351-239-835295.

E-mail address: brett@ci.uc.pt (A.M. Oliveira-Brett).
}

further purification. Solutions of different concentration of purine bases were prepared in buffer electrolyte solutions of ionic strength $(0.1)$ using analytical grade reagents and purified water from Millipore Milli-Q system (conductivity $\left.<0.1 \mu \mathrm{S} \mathrm{cm}^{-1}\right)$. All voltammetric experiments were done using a $\mu$ Autolab running with GPES version 4.8 software, Eco-Chemie, Utrecht, Netherlands. A one-compartment electrochemical cell with a volumetric capacity of $1 \mathrm{ml}$ was used containing a $7-\mu \mathrm{m}$ diameter glassy carbon

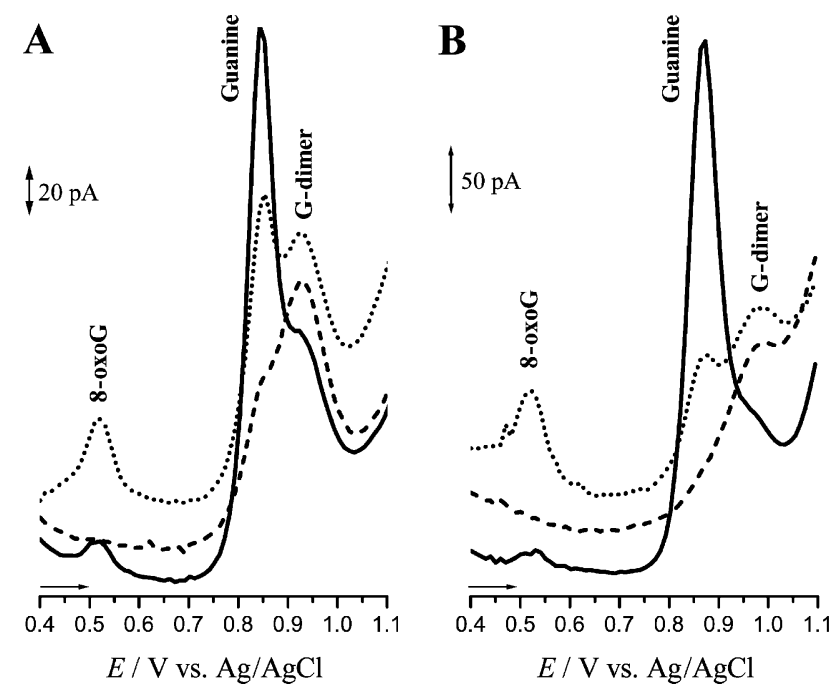

Fig. 1. Differential pulse voltammograms of guanine, 5 th scan (-) (A) 0.5 $\mathrm{mM}$ and (B) $50 \mu \mathrm{M}$, in $\mathrm{pH} 4.50 .2 \mathrm{M}$ acetate buffer at a glassy carbon microelectrode. After transferring the microelectrode to supporting electro-

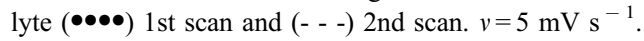



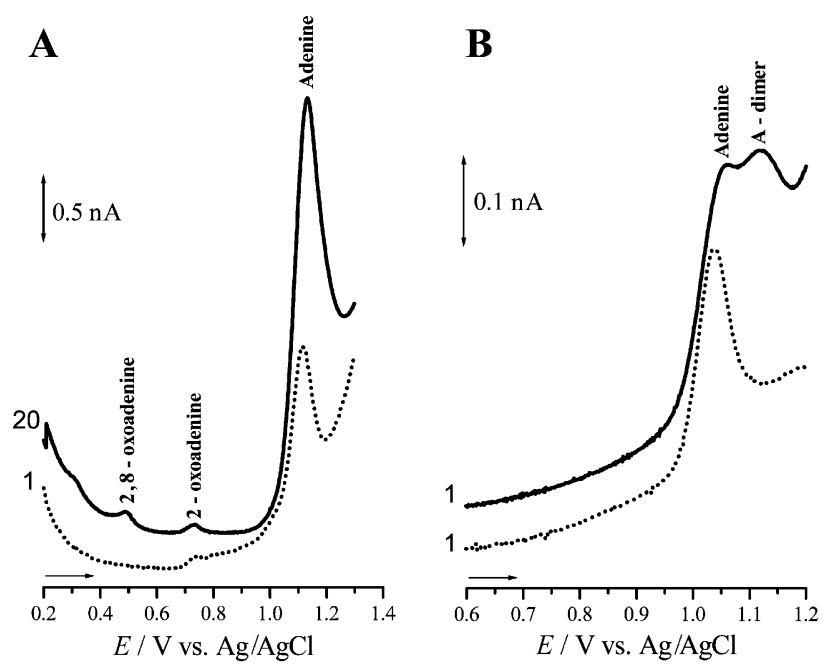

Fig. 2. Differential pulse voltammograms of adenine (-) (A) $1 \mathrm{mM} 20$ th scan and (B) $10 \mu \mathrm{M} 1 \mathrm{st}$ scan, in $\mathrm{pH} 4.50 .2 \mathrm{M}$ acetate buffer at a glassy carbon microelectrode. After transferring the microelectrode to supporting electrolyte $(\bullet \bullet \bullet)) 1$ st scan. $v=5 \mathrm{mV} \mathrm{s}^{-1}$.

microelectrode, Cypress, USA, a Pt wire counter electrode, and an $\mathrm{Ag} / \mathrm{AgCl}$ reference electrode. The cell was placed inside a Faraday cage at room temperature during all the measurements. The experimental conditions were: cyclic voltammetry, scan rate $3 \mathrm{~V} \mathrm{~s}^{-1}$; differential pulse voltammetry, pulse amplitude $50 \mathrm{mV}$, pulse width $70 \mathrm{~ms}$, scan rate $5 \mathrm{mV} \mathrm{s}^{-1}$.

\section{Results and discussion}

Guanine oxidation is irreversible and occurs in two consecutive steps, Fig. 1 . The first step is guanine oxidation to 8-oxoguanine (8-oxoG) and, at $\mathrm{pH} 4.5$, occurs at $+0.8 \mathrm{~V}$ vs. $\mathrm{Ag} / \mathrm{AgCl}$, and is a two-electron/two-proton irreversible process [4] and the peak at $+0.95 \mathrm{~V}$ corresponds to the oneelectron transfer reversible oxidation of the guanine dimers (G-dimer) [2,3]. The peak at $+0.55 \mathrm{~V}$ corresponding to the reversible two-electron/two-proton oxidation of 8-oxoG, formed on the electrode surface, is clearly observed after five scans. The microelectrode was transferred to supporting electrolyte after these experiments and all three peaks appeared in the first scan. The first two peaks disappeared in subsequent scans because all the guanine and 8-oxoG adsorbed on the electrode surface was oxidised, and oxidised 8-oxoG undergoes rapid hydrolysis [4]. The third peak remains because it corresponds to the reversible oxidation of the strongly adsorbed G-dimers formed during the oxidation of guanine.
Adenine oxidation is also irreversible, and occurs in three steps [5,6]; and for millimolar concentrations, these peaks could be identified by fast cyclic voltammetry, $3 \mathrm{~V} \mathrm{~s}^{-1}$ [6]. However, the sharper adenine oxidation peak found at 1$\mathrm{mM}$ concentrations splits into two peaks for $10 \mu \mathrm{M}$ and another electrode processes was identified by differential pulse voltammetry, Fig. 2 . The first peak, at $+1.05 \mathrm{~V}$, corresponds to adenine oxidation and the second, at $+1.12 \mathrm{~V}$, to the oxidation of adenine dimers. The electroactive adenine oxidation products 2,8-oxoadenine and 2-oxoadenine formed on the electrode surface are detected after 20 scans. Adenine adsorbs very strongly on the electrode surface, compared to the oxidation products and the dimers, and the oxidation peak is observed after transferring the electrode to the supporting electrolyte.

\section{Conclusion}

The use of microelectrodes and of low concentrations of guanine and adenine enabled a more detailed study of the electrochemical oxidation for both purines. It occurs in consecutive steps, with the formation of dimers.

\section{Acknowledgements}

Financial support from Fundação para a Ciência e Tecnologia (FCT) project PRAXIS/C/SAU/93/96, POCTI (co-financed by the European Community Fund FEDER) ICEMS (Research Unit 103) and European Projects ERBICT15-CT960804 and QLK3-2000-01311, are gratefully acknowledged.

\section{References}

[1] A.M. Oliveira-Brett, S.H.P. Serrano, J.A.P. Piedade, Electrochemistry of DNA, in: R.G. Compton, G. Hancock (Eds.), Comprehensive Chemical Kinetics, vol. 37, Elsevier, Amsterdam, 1999, pp. 91-119, cap. 3.

[2] R.N. Goyal, G. Dryhurst, Redox chemistry of guanine and 8-oxyguanine and a comparison of the peroxidase-catalysed and electrochemical oxidation of 8-oxyguanine, J. Electroanal. Chem. 135 (1982) 75-91.

[3] P. Subramanian, G. Dryhurst, Electrochemical oxidation of guanosine, Formation of some novel guanine oligonucleosides, J. Electroanal. Chem. 244 (1987) 137-162.

[4] A.M. Oliveira-Brett, J.A.P. Piedade, S.H.P. Serrano, Electrochemical oxidation of 8-oxoguanine, Electroanalysis 12 (2000) 969-973.

[5] G. Druyhurst, Dicarbonium ions as products of electrochemical oxidation of biologically important purines at the pyrolytic graphite electrode, J. Electrochem. Soc. 116 (1969) 1411-1412.

[6] G. Druyhurst, P.J. Elving, Electrochemical oxidation of adenine: reaction products and mechanisms, J. Electrochem. Soc. 115 (1968) 10141020 